\title{
Genetic control observation for yield and morphological traits in maize (Zea mays L.) inbred lines
}

\begin{abstract}
Forty crosses produced from 20 lines and 2 testers (CL02450 and CML 451) using Line $\times$ Tester mating design along with 3 standard checks (BHM9, 981 and Sunshine) were evaluated following alpha lattice design to find better hybrid(s). Significant variation was observed among the hybrids (crosses). Variance due to specific combining ability (SCA) was larger than that due to general combining ability (GCA) for the all characters indicating the preponderance of non-additive gene action in the expression of various traits. Among the lines, CML430, CML429 and CML223 and one tester CML451 were good general combiners for grain yield and some of the important yield-contributing characters. Cross Hybrids like CML $287 \times$ CML451 and CML431 $\times$ CLO2450 showed significant positive SCA effects and heterosis for grain yield along with the highest mean could be used for variety development.
\end{abstract}

Volume 8 Issue 5 - 2018

\author{
AH Akhi, SA Ahmed, ANMS Karim, MM \\ Rohman
}

Plant Breeding Division, Bangladesh Agricultural Research Institute, Bangladesh

Correspondence: MM Rohman, Plant Breeding Division, Bangladesh Agricultural Research Institute, Gazipur, Bangladesh, Email motiar_I@yahoo.com

Received: March 08, 2018| Published: September 04, 2018

Keywords: diallel, combining ability, heterosis, maize

\section{Introduction}

Maize grain is recognized worldwide as a strategic food and feed crop. Maize grains provide an enormous amount of protein and energy for humans and livestock like quality protein maize. ${ }^{1}$ Because of being highly allogamous crop, maize has been successfully exploited for the production of hybrids. In meeting the increasing world population maize (Zea mays L.) hybrid development is a foremost factor during the past 40 years. For getting advantageous genetic information, combining ability is a powerful tool for selection of desirable parents depends on their performance in a line of crosses. Line $\mathrm{x}$ tester is useful in deciding the relative ability of female and male lines to produce desirable hybrid combinations. ${ }^{2}$ It empowers the breeder for choosing convenient method for hybrid variety development. Knowledge on combining ability effects helps the breeder in choosing the parents and hybrids with the high general combining ability and specific combining, respectively. Combining ability analysis (general combining ability (GCA), specific combining ability (SCA) and heterosis analysis) helps in identifying inherent inbred lines for development synthetic and hybrids varieties in maize breeding program. The goal of the existing study was to observe the combining ability (GCA and SCA) for different traits in the studied material.

\section{Materials and methods}

\section{Experimental materials}

The materials for this experiment consisted of 20 newly developed and selected inbred lines of field corn were crossed with two tester's viz., CL02450 and CML 451 during Rabi, 2015-16 at Bangladesh Agricultural Research Institute, Gazipur, and $40 \mathrm{~F}_{1}$ hybrids were produced. Subsequently, during Rabi, 2016-17 these $40 \mathrm{~F}_{1}$ crosses along with 3 standard checks (BARI Hybrid Maize 9, 981 and Sunshine) and all the parents (lines and testers) were evaluated.

\section{Experimental design and field managements}

The experimental design used for the field evaluation was alpha lattice design ${ }^{3}$ with two replications. Each entry consisted of one row of $4 \mathrm{~m}$ long and spacing was $60 \mathrm{~cm}$ between rows and $25 \mathrm{~cm}$ between plants within the row. Fertilizers were applied @250, 120, 120, 40,
5 and $1 \mathrm{~kg} /$ ha of N, P, K, S, Zn and B, respectively. Per hill two seed were planted to confirm enough plant stands in the field. At three to five-leaf stages proper thinning was done and in each hill one healthy plant was kept. Undesirable and off type plants from each line were rouged out before flowering. Standard agronomic practices were followed to raise a good crop.

\section{Procedures and statistical analysis}

Data collection and analysis of variance (ANOVA): Data were recorded on eight quantitative characters. Data related to days to $50 \%$ pollen shedding, $50 \%$ days to silking and grain yield were recorded on the plot basis while data related to other characters viz, plant height, ear height, ear length were recorded on five randomly selected plants and number of grains per row and 1000-grain weight were recorded on five randomly selected ears leaving border plants of each row. The mean values were subjected to line $\mathrm{x}$ tester analysis. Analyses of variances (ANOVA) were computed for grain yield and other agronomic traits by using CROP Stat 7.2 software.

Combining ability analysis: Line $\mathrm{x}$ tester analysis was done for traits that showed statistically significant differences among the crosses applying the adjusted means following the method described by Kempthorne. ${ }^{2}$ General combining ability (GCA) and specific combining ability (SCA) effects for grain yield and other agronomic traits were calculated using the line $\mathrm{x}$ tester model. The F-test of mean square due to lines, testers and their interactions were computed against mean square due to error Singh. ${ }^{4}$ Significances of GCA and SCA effects of the lines and hybrids were determined by an F-test using the standard errors of GCA and SCA effects.

Heterosis analysis: Percent heterosis was calculated by using the following formula:

Standard heterosis $(\%)=\left[\left(\mathrm{F}_{1}-\mathrm{CV}\right) / \mathrm{CV}\right] \times 100$

Where, $\mathrm{F}_{1}$ and $\mathrm{CV}$ represented the mean performance of hybrid and standard check variety.

The significance test for heterosis was done by using standard error of the value of check variety. 


\section{Results and discussion}

Analysis of variance for combining ability was carried out for yield and yield contributing characters and the mean sum of squares are presented in Table 1. The mean squares due to the parents, parents vs. crosses, crosses, lines, testers, and line $\mathrm{x}$ tester were found to be significant for most of the characters under study indicating presence of genetic variability among the genotypes for those characters. When the effects of crosses partitioned into lines, testers and line $\times$ tester interaction effects, the interaction effects (line $\times$ testers) were found to be highly significant for ear length, number of grains per row and for 1000-grain weight indicating that hybrids differed significantly in their SCA effects. The variances for line were found to be significant for all of the characters but variances due to tester were found to be significant only for plant height. Significant variances due to lines for different traits suggest that these traits were predominantly controlled by additive type of gene action.

Table I Mean squares and estimates of variance for grain yield, yield components and other characters in maize evaluated at Gazipur during Rabi 20 I6-I7

\begin{tabular}{|c|c|c|c|c|c|c|c|c|c|}
\hline Sources & df & $\begin{array}{l}\text { DPS } \\
\text { (day) }\end{array}$ & $\begin{array}{l}\text { DS } \\
\text { (day) }\end{array}$ & $\begin{array}{l}\text { PH } \\
(\mathrm{cm})\end{array}$ & $\begin{array}{l}\text { EH } \\
(\mathrm{cm})\end{array}$ & $\begin{array}{l}\text { EL } \\
(\mathrm{cm})\end{array}$ & $\begin{array}{l}\text { NGPR } \\
\text { (no.) }\end{array}$ & $\begin{array}{l}\text { TGW } \\
\text { (g) }\end{array}$ & $\mathbf{Y}(\mathrm{t} / \mathrm{ha})$ \\
\hline Genotypes & 61 & $76.17^{* *}$ & $77.89 * *$ & $795.50 * *$ & $450.53^{* *}$ & $9.62 * *$ & $82.50 * *$ & $4551.24 * *$ & $13.600 * *$ \\
\hline Parents (P) & 21 & $90.88 * *$ & $91.65^{* *}$ & $648.84 * *$ & $408.49 * *$ & $8.4 I^{* * *}$ & $92.55^{* *}$ & $3380.62 * *$ & $4.416 * *$ \\
\hline$P$ vs $C$ & I & $453.42 * *$ & $514.66 * *$ & $|863| .86^{* *}$ & $8758.02^{* *}$ & $235.19 * *$ & $1818.32 * *$ & $26038.45^{* *}$ & $507.19 * *$ \\
\hline Crosses(C) & 39 & $58.57 * *$ & $59.29 * *$ & $417.13^{* *}$ & $260.15^{* *}$ & $4.48^{* * *}$ & $32.59 * *$ & $4630.62 * *$ & $5.89 * *$ \\
\hline Lines & 19 & $87.17^{* *}$ & $87.38 * *$ & $501.37 * *$ & $331.69 * *$ & $3.48^{* *}$ & $33.56 * *$ & $4615.16 * *$ & $7.13 * *$ \\
\hline Testers & I & 2.81 & 4.51 & $|665.3|^{* *}$ & 234.61 & 3.61 & 30.01 & 2080.8 & 2.54 \\
\hline LxT & 19 & 32.92 & 34.09 & 267.21 & 189.95 & $5.53^{* *}$ & $31.75^{* * *}$ & $4780.27 * *$ & 4.82 \\
\hline Error & 61 & 21.54 & 22.17 & 130.58 & 134.03 & 0.98 & 10.17 & 855.133 & 3.29 \\
\hline \multicolumn{10}{|c|}{ Estimate of component of variance } \\
\hline$\sigma 2 g$ (line) & & 13.56 & 13.32 & 58.59 & 35.43 & 0.51 & 0.45 & 41.28 & 0.58 \\
\hline$\sigma 2 g$ (tester) & & 0.75 & 0.74 & 34.95 & 1.12 & 0.04 & 0.04 & 67.49 & 0.057 \\
\hline$\sigma 2 g c a$ & & 0.43 & 0.42 & 2.52 & 1.18 & 0.02 & 0.01 & 2.52 & 0.018 \\
\hline$\sigma 2 s c a$ & & 5.68 & 5.96 & 63.32 & 27.95 & 2.28 & 10.79 & 1962.57 & 0.77 \\
\hline$\sigma 2 \mathrm{gca} / \sigma 2 \mathrm{sca}$ & & 0.07 & 0.07 & 0.03 & 0.04 & 0.008 & 0.001 & 0.001 & 0.02 \\
\hline
\end{tabular}

*Significant at $5 \%$ level, **Significant at I\% level

DPS, days to pollen shed; DS, days to silk; PH, plant height; EH, ear height; EL, ear length; NGPR, number of grains per row; TGW, I000- grain weight; Y, yield

This outcome is similar to previous findings by Yazachew et al., 5 who reported that non-additive gene action to be more important than additive gene effects for these traits The ratio of GCA and SCA variance for all the traits under study viz., days to 50 percent pollen shedding (0.07), days to 50 per cent silking (0.07), plant height $(0.03)$, ear height (0.04), ear length (0.008), number of grains per row $(0.008)$, 1000 -grain weight $(0.001)$ and yield $(0.02)$ were less than one, which indicated that all these characters were predominantly governed by non-additive gene effects (Table 1). Similar findings were reported by Kanagarasu et al., ${ }^{6}$ and Kumar et al., ${ }^{7}$ for grain yield, cob length, plant height, ear height, 100 grain weight, grain rows per cob, days to 50 per cent tassel and days to 50 per cent silk and Ali et al., ${ }^{8}$ for number of grain rows per cob and 100-grain weight and Kumar et al., ${ }^{9}$ for plant height, days to $50 \%$ tassel, days to $50 \%$ silk, cob length, cob girth, number of grain rows per cob, number of grains per row, 100-grain weight and grain yield in maize in their study. The contribution of lines, testers and interactions to total variance are presented in Table
2. The proportional contribution of lines to the total variances was much higher than tester in all the traits indicating higher estimates of variances due to general combining ability. Results showed that maternal parents play the most important role for those traits. Similar conclusion was reported by Amiruzzaman ${ }^{10}$ who observed the greater effect of female lines for grain yield, kernel weight and kernel number in maize.

\section{General combining ability (GCA) effects}

The general combining ability (GCA) effects of lines (females) and testers (males) are presented in Table 3. Significant and negative GCA were expected for days to tassel as well as silk, plant height and ear height to produce early and dwarf hybrid. And for yield and yieldrelated traits positive GCA effects were expected for high yielding commercial hybrid. The estimate of GCA showed that among the lines only one line CML427 had significant and negative GCA effect for days to pollen shedding indicating earliness. Lines CML425, BIL77, 
and CML465 showed significant negative GCA effects for plant height. Among these three lines BIL77 had the significant negative GCA effect for both plant height and ear height indicating this line was good general combiner for dwarfness. Bhavana et al., ${ }^{11}$ and Jawaharlal et al., ${ }^{12}$ also reported the additive gene action for days to 50 percent tassel and silk. BIL 79 exhibited significant and positive GCA effect for ear length which could contribute for evolving longer ears. CLG1837, CL02720, CML496, CML473, CML486 was observed to be good combiner for number of grain per row and CML429, CML430 and CML223 for thousand grain weight because these line showed significant and positive GCA effect. Between the testers CML451 were observed best combiner for grain yield. Among the lines only one line CML 430 were found to be the best combiner for grain yield (Table 3). This line could be extensively utilized for evolving high yields. Significant GCA effect for yield in maize was also reported by Amin et al., ${ }^{13}$; Kumar et al., ${ }^{7} ;$ Ivy \& Hawlader ${ }^{14}$ and Amiruzzaman. ${ }^{10}$

Table 2 Proportional contribution of lines, testers and their interactions to total variance in maize

\begin{tabular}{|c|c|c|c|c|c|c|c|c|}
\hline Source & $\begin{array}{l}\text { DPS } \\
\text { (day) }\end{array}$ & DS (day) & $\mathrm{PH}(\mathrm{cm})$ & $\begin{array}{l}\mathrm{EH} \\
(\mathrm{cm})\end{array}$ & $\begin{array}{l}\text { EL } \\
(\mathrm{cm})\end{array}$ & NGPR (no.) & $\begin{array}{c}\text { TGW } \\
\text { (g) }\end{array}$ & $\begin{array}{l}Y \\
\text { t/ha) }\end{array}$ \\
\hline Line $(L)$ & 72.49 & 71.79 & 58.56 & 62.11 & 37.81 & 50.17 & 48.56 & 59.01 \\
\hline Tester $(T)$ & 0.12 & 0.19 & 10.24 & 2.31 & 2.06 & 2.36 & 1.15 & 1.1 \\
\hline LXT & 27.38 & 28.01 & 31.2 & 35.57 & 60.11 & 47.47 & 50.29 & 39.88 \\
\hline
\end{tabular}

DPS, days to pollen shedding; DS, days to silk; PH, plant height; EH, ear height; EL, ear length, NGPR, number of grains per row; TGW, I000- grain weight; Y, yield Table 3 General combining ability (GCA) effects and mean of parents for grain yield and yield components and other characters in maize

\begin{tabular}{|c|c|c|c|c|c|c|c|c|c|c|c|c|c|c|c|c|}
\hline \multirow[t]{2}{*}{ Parents } & \multicolumn{2}{|c|}{ DPS (day) } & \multicolumn{2}{|l|}{ DS (day) } & \multicolumn{2}{|l|}{$\mathrm{PH}(\mathrm{cm})$} & \multicolumn{2}{|l|}{$\mathrm{EH}(\mathrm{cm})$} & \multicolumn{2}{|l|}{$\mathrm{EL}(\mathrm{cm})$} & \multicolumn{2}{|c|}{ NGPR (no.) } & \multicolumn{2}{|c|}{ TGW (gm) } & \multicolumn{2}{|l|}{$Y(t / h a)$} \\
\hline & GCA & mean & GCA & mean & GCA & mean & GCA & mean & GCA & mean & GCA & mean & GCA & mean & GCA & mean \\
\hline \multicolumn{17}{|l|}{ Testers } \\
\hline I. CL02450 & -0.18 & 99 & -0.24 & 101 & $-4.68 *$ & 154 & -1.71 & 70 & -0.21 & 12 & -0.61 & 26 & -4.8 & 260 & $-1.07 * *$ & 2.6 \\
\hline 2. CML $45 \mathrm{I}$ & 0.18 & 92 & 0.24 & 96 & $4.68^{*}$ & 111 & 1.71 & 33 & 0.21 & 13 & 0.61 & 19 & 4.8 & 215 & $1.07 * *$ & 6.3 \\
\hline $\mathrm{SE}(\mathrm{gi})$ & 0.73 & & 0.74 & & 1.81 & & 1.83 & & 0.16 & & 0.5 & & 4.62 & & 0.29 & \\
\hline $\mathrm{SE}(\mathrm{gi}-\mathrm{g} \mathrm{j})$ & 1.03 & & 1.05 & & 2.56 & & 2.56 & & 0.22 & & 0.71 & & 6.54 & & 0.41 & \\
\hline \multicolumn{17}{|l|}{ Lines } \\
\hline I.CLG 1837 & -3.66 & 97 & -3.54 & 100 & 0.56 & 98 & 7.41 & 26 & 0.96 & 9 & $3.84^{*}$ & 20 & $-36.65^{*}$ & 255 & 1.5 & 2.2 \\
\hline 2.CL02720 & 1.34 & 93 & 1.46 & 97 & -0.94 & 142 & 1.91 & 67 & 0.71 & 12 & $4.09 * *$ & 22 & 8.35 & 245 & 1.12 & 3.5 \\
\hline 3.CML285 & -0.41 & 111 & -0.54 & 114 & 7.56 & 125 & 5.16 & 46 & -0.04 & 8 & -1.41 & 17 & -4.15 & 223 & 1 & 2.7 \\
\hline 4.CML 287 & -2.16 & 93 & -2.29 & 95 & $13.56^{*}$ & 164 & $13.4 \mid$ & 66 & -0.04 & 16 & 0.09 & 31 & -14.15 & 315 & -0.6 & 6.2 \\
\hline 5.CML298 & -2.16 & 93 & -2.04 & 95 & $16.06 * *$ & 116 & 12.91 & 48 & 0.21 & 14 & 1.09 & 31 & -6.65 & 310 & 1.52 & 4.6 \\
\hline 6.CML425 & -1.91 & 82 & -2.04 & 85 & $-23.69 * *$ & 130 & -14.3 & 50 & 0.21 & 14 & 1.09 & 20 & 5.85 & 223 & -0.03 & 5 \\
\hline 7.CML429 & 1.09 & 87 & 0.96 & 90 & 5.31 & 119 & $12.66^{*}$ & 38 & -0.04 & 12 & $-3.16 *$ & 15 & $40.85 * *$ & 235 & 1.01 & 1.1 \\
\hline 8.CML430 & -1.16 & 88 & -1.04 & 91 & 4.31 & 160 & 6.41 & 69 & 0.46 & 14 & $-0.4 I$ & 34 & $55.85 * *$ & 340 & $1.21 * *$ & 6.4 \\
\hline 9.CML43I & 0.59 & 93 & -0.54 & 96 & $12.31 *$ & 121 & 6.66 & 60 & 0.71 & 14 & 0.84 & 34 & 3.35 & 260 & 0.54 & 3.6 \\
\hline 10.CML486 & -2.16 & 94 & -2.54 & 96 & -6.19 & 149 & 5.41 & 65 & 0.46 & 13 & $4.34 * *$ & 22 & 10.85 & 300 & -0.82 & 3.3 \\
\hline II.CML223 & $11.59 * *$ & 95 & 11.96 ** & 99 & -6.19 & 116 & -12.3 & 38 & -0.79 & 11 & -0.91 & 9 & $40.85 * *$ & 210 & 0.23 & 3.2 \\
\hline I2.BIL79 & -1.16 & 90 & -1.54 & 93 & 7.56 & 144 & 0.91 & 63 & $\left.1.2\right|^{*}$ & 14 & -0.91 & 30 & 25.85 & 255 & -0.65 & 6 \\
\hline |3.BIL77 & -0.41 & 92 & -0.29 & 95 & $-18.69 * *$ & 125 & $-14.59 *$ & 57 & -0.79 & 12 & -3.41 & 20 & -16.65 & 290 & -0.37 & 3.1 \\
\hline I4.CML427 & $-4.66 *$ & 106 & -4.29 & 109 & -8.94 & 115 & -8.34 & 23 & $-3.04 * *$ & 8 & $-4.16 * *$ & 11 & $-34.15^{*}$ & 300 & $-3.91 * *$ & 1.8 \\
\hline \multicolumn{17}{|l|}{ Table 3 cont'd } \\
\hline & \multicolumn{2}{|c|}{ DPS (day) } & \multicolumn{2}{|l|}{ DS (day) } & \multicolumn{2}{|l|}{$\mathrm{PH}(\mathrm{cm})$} & \multicolumn{2}{|l|}{$\mathrm{EH}(\mathrm{cm} 0$} & \multicolumn{2}{|l|}{$\mathrm{EL}(\mathrm{cm})$} & \multicolumn{2}{|c|}{ NGPR (no.) } & \multicolumn{2}{|c|}{ TGW (gm) } & \multicolumn{2}{|l|}{$Y(t / h a)$} \\
\hline Parents & GCA & mean & GCA & mean & GCA & mean & GCA & mean & GCA & mean & GCA & mean & GCA & mean & GCA & mean \\
\hline I5.CML473 & -1.91 & 108 & -1.79 & 110 & 8.06 & 127 & -3.84 & 38 & -0.04 & 10 & $3.84 *$ & 26 & 30.85 & 335 & -0.1 & 4.3 \\
\hline 16.VLI016220 & -2.66 & 90 & -2.29 & 93 & -4.44 & 144 & -7.84 & 60 & -0.79 & 14 & $-4.16 * *$ & 24 & $-54.15^{* *}$ & 220 & -1.8 & 3.8 \\
\hline 17.CML465 & 0.09 & 94 & 0.46 & 99 & $-15.19 *$ & 136 & -9.59 & 59 & -0.54 & 14 & $-4.4 I^{* *}$ & 28 & $-68.65 * *$ & 295 & -1.64 & 4.8 \\
\hline
\end{tabular}


Table Continued

\begin{tabular}{|c|c|c|c|c|c|c|c|c|c|c|c|c|c|c|c|c|}
\hline \multirow[t]{2}{*}{ Parents } & \multicolumn{2}{|c|}{ DPS (day) } & \multicolumn{2}{|c|}{ DS (day) } & \multicolumn{2}{|c|}{$\mathrm{PH}(\mathrm{cm})$} & \multicolumn{2}{|c|}{$\mathrm{EH}(\mathrm{cm})$} & \multicolumn{2}{|c|}{ EL $(\mathrm{cm})$} & \multicolumn{2}{|c|}{ NGPR (no.) } & \multicolumn{2}{|c|}{ TGW (gm) } & \multicolumn{2}{|c|}{$Y(t / h a)$} \\
\hline & GCA & mean & GCA & mean & GCA & mean & GCA & mean & GCA & mean & GCA & mean & GCA & mean & GCA & mean \\
\hline |8.CML48| & $14.09 * *$ & 93 & 13.96 ** & 95 & -8.94 & 112 & -6.84 & 41 & 0.46 & 10 & -0.66 & 26 & 20.85 & 270 & 0.65 & 2.8 \\
\hline 19.CML496 & -3.16 & 98 & -3.04 & 101 & 10.31 & 143 & 1.66 & 60 & -0.29 & 12 & $3.09 *$ & 18 & $-39.15 * *$ & 285 & 0.2 & 3.5 \\
\hline 20.CML433 & -1.16 & 89 & -1.04 & 93 & 7.56 & 154 & 3.16 & 61 & 0.96 & 13 & 1.34 & 25 & 30.85 & & 1.29 & 4.8 \\
\hline SE(gi) & 2.32 & & 2.35 & & 5.71 & & 5.79 & & 0.49 & & 1.59 & & 14.62 & & 0.44 & \\
\hline SE(gi-gj) & 3.23 & & 3.33 & & 8.08 & & 3.19 & & 0.7 & & 2.26 & & 20.68 & & 1.28 & \\
\hline
\end{tabular}

*Significant at $5 \%$ level, **Significant at $1 \%$ level

DPS, days to pollen shedding; DS, days to silk; PH, plant height; EH, ear height; EL, Ear length; NGPR, number of grains per row; TGW, I000- grain weight ;Y, yield

\section{Specific combining ability (SCA) effects}

The specific combining ability effects are presented in the Table 4. Among 40 crosses, many of the crosses were ranked as top crosses for one or more characters. None of the crosses was found desirable simultaneously for all the characters i.e., different crosses expressed significant SCA effects for different characters. Among the 40 cross combinations, only one cross CML287xCML451 had significant and positive SCA effect for grain yield. And another cross CML431xCLO2450 exhibited significant positive SCA effect of both number of grain per row and 1000 grain weight with the highest mean for yield. Nadarajan and Gunsekarn ${ }^{15}$ explained that the cross combinations with non-significant SCA effects (absence of dominance) as well as parents with significant GCA effects (presence of additive gene action) could be useful in identification of superior segregates. The crosses which exhibited significant desirable SCA effects were CLG $1837 \mathrm{xCML} 451$ for tasseling and silking, CLG 1837 XCLO2450 for plant height and none of the plant showed significant negative SCA effect for ear height. Similarly while considering cob and grain characters, the crosses viz., CL02720 xCML451, CML287xCML451, CML431xCML451 (eear length), CL02720xCLO2450, CML430xCLO2450, CML431xCLO2450 (no of grain per row) expressed significant positive SCA effects. Kanagarasu et al., ${ }^{6}$ find similar type of result in maize.

Table 4 Specific combining ability (SCA) effects and mean of the crosses for grain yield and yield contributing characters in maize

\begin{tabular}{|c|c|c|c|c|c|c|c|c|c|c|c|c|c|c|c|c|}
\hline \multirow[t]{2}{*}{ Parents } & \multicolumn{2}{|c|}{ DPS (day) } & \multicolumn{2}{|c|}{ DS (day) } & \multicolumn{2}{|l|}{ PH (cm) } & \multicolumn{2}{|c|}{ EH (cm) } & \multicolumn{2}{|c|}{ EL (cm) } & \multicolumn{2}{|c|}{ NGPR (no.) } & \multicolumn{2}{|c|}{ TGW (gm) } & \multicolumn{2}{|c|}{$\mathbf{Y}(\mathbf{t} / \mathbf{h a})$} \\
\hline & SCA & mean & SCA & mean & SCA & mean & SCA & mean & SCA & mean & SCA & mean & SCA & mean & SCA & mean \\
\hline $\begin{array}{l}\text { 1.CLG } 1837 \mathrm{X} \\
\text { CLO2450 }\end{array}$ & -0.81 & 86 & -0.76 & 89 & $-19.44 * *$ & 138 & -13.8 & 61 & -0.54 & 15 & $-5.14 * *$ & 29 & $-4.90^{* *}$ & 255 & -1.07 & 8.4 \\
\hline $\begin{array}{l}\text { 2.CL02720 X } \\
\text { CLO2450 }\end{array}$ & 0.81 & 88 & 0.76 & 91 & $19.44 * *$ & 185 & 13.79 & 92 & 0.54 & 17 & $5.14 * *$ & 41 & $4.90^{* *}$ & 275 & 1.07 & 10.9 \\
\hline $\begin{array}{l}\text { 3.CML285 X } \\
\text { CLO2450 }\end{array}$ & 1.19 & 93 & 1.24 & 96 & 4.06 & 160 & -1.29 & 68 & 1.21 & 17 & 2.11 & 37 & $55.10^{* *}$ & 360 & 1.37 & 10.4 \\
\hline $\begin{array}{l}\text { 4.CML } 287 \mathrm{X} \\
\text { CLO2450 }\end{array}$ & -1.19 & 91 & -1.24 & 94 & -4.06 & 163 & 1.29 & 74 & -1.21 & 15 & -2.11 & 34 & $-55.10^{* *}$ & 260 & -1.37 & 8 \\
\hline $\begin{array}{l}\text { 5.CML298 X } \\
\text { CLO2450 }\end{array}$ & -1.06 & 89 & -1.26 & 91 & 7.06 & 169 & 9.46 & 82 & 0.46 & 15 & 1.11 & 30 & $-47.40^{* *}$ & 245 & 0.95 & 9.9 \\
\hline $\begin{array}{l}\text { 6.CML425 X } \\
\text { CLO2450 }\end{array}$ & 1.06 & 91 & 1.26 & 94 & -7.06 & 170 & -9.46 & 67 & -0.46 & 15 & -1.11 & 29 & $47.40^{* *}$ & 350 & -0.95 & 8.3 \\
\hline $\begin{array}{l}\text { 7.CML429 X } \\
\text { CLO2450 }\end{array}$ & -2.81 & 85 & -2.51 & 88 & -5.94 & 177 & -10.3 & 71 & -1.54 & 13 & $-5.89 * *$ & 25 & $-27.40^{* *}$ & 255 & -0.44 & 6.9 \\
\hline $\begin{array}{l}\text { 8.CML430 X } \\
\text { CLO2450 }\end{array}$ & 2.81 & 91 & 2.51 & 94 & 5.94 & 180 & 10.29 & 95 & 1.54 & 17 & $5.89 * *$ & 38 & $27.40^{* *}$ & 320 & 0.44 & 8.1 \\
\hline $\begin{array}{l}\text { 9.CML431 X } \\
\text { CLO2450 }\end{array}$ & -0.81 & 87 & -0.76 & 90 & -0.44 & 177 & 3.21 & 84 & 0.71 & 16 & $6.11^{* *}$ & 38 & $5.10^{* *}$ & 295 & 1.96 & 11.4 \\
\hline $\begin{array}{l}\text { 10.CML486 X } \\
\text { CLO2450 }\end{array}$ & 0.81 & 89 & 0.76 & 92 & 0.44 & 173 & -3.21 & 81 & -0.71 & 15 & $-6.11 * *$ & 27 & $-5.10^{* *}$ & 295 & -1.96 & 7.9 \\
\hline $\begin{array}{l}\text { 11.CML223 X } \\
\text { CLO2450 }\end{array}$ & -0.06 & 88 & -0.26 & 91 & 0.31 & 134 & 6.96 & 60 & -0.29 & 15 & -0.89 & 31 & $17.60^{* *}$ & 320 & 0.71 & 8.6 \\
\hline $\begin{array}{l}\text { 12.BIL79 X } \\
\text { CLO2450 }\end{array}$ & 0.06 & 89 & 0.26 & 92 & -0.31 & 145 & -6.96 & 50 & 0.29 & 16 & 0.89 & 34 & $-17.60^{* *}$ & 295 & -0.71 & 7.6 \\
\hline $\begin{array}{l}\text { 13.BIL77 X } \\
\text { CLO2450 }\end{array}$ & -5.06 & 86 & -5.26 & 89 & 10.81 & 178 & 10.96 & 91 & -0.04 & 15 & -1.64 & 26 & $-22.40^{* *}$ & 315 & 0.26 & 9.2 \\
\hline $\begin{array}{l}\text { 14.CML427 X } \\
\text { CLO2450 }\end{array}$ & 5.06 & 97 & 5.26 & 100 & -10.81 & 150 & -11 & 73 & 0.04 & 15 & 1.64 & 30 & $22.40^{* *}$ & 370 & -0.26 & 9.1 \\
\hline $\begin{array}{l}\text { 15.CML473 X } \\
\text { CLO2450 }\end{array}$ & 4.19 & 93 & 4.24 & 96 & -6.69 & 147 & -7.29 & 67 & -0.54 & 15 & 0.11 & 30 & $12.60^{* *}$ & 365 & -1.25 & 7.6 \\
\hline $\begin{array}{l}\text { 16.VL1016220 X } \\
\text { CLO2450 }\end{array}$ & -4.19 & 85 & -4.24 & 88 & 6.69 & 177 & 7.29 & 85 & 0.54 & 16 & -0.11 & 31 & $-12.60^{* *}$ & 350 & 1.25 & 10.4 \\
\hline $\begin{array}{l}\text { 17.CML } 465 \mathrm{X} \\
\text { CLO2450 }\end{array}$ & -2.06 & 89 & -1.76 & 91 & 5.31 & 165 & -6.04 & 68 & 1.21 & 17 & 1.86 & 33 & $-54.90 * *$ & 245 & 0.87 & 9.3 \\
\hline
\end{tabular}


Table Continued

\begin{tabular}{|c|c|c|c|c|c|c|c|c|c|c|c|c|c|c|c|c|}
\hline \multirow[t]{2}{*}{ Parents } & \multicolumn{2}{|l|}{ DPS (day) } & \multicolumn{2}{|l|}{ DS (day) } & \multicolumn{2}{|l|}{ PH (cm) } & \multicolumn{2}{|l|}{ EH (cm) } & \multicolumn{2}{|l|}{ EL (cm) } & \multicolumn{2}{|c|}{ NGPR (no.) } & \multicolumn{2}{|l|}{ TGW (gm) } & \multicolumn{2}{|l|}{$\mathbf{Y}(\mathbf{t} / \mathbf{h a})$} \\
\hline & SCA & mean & SCA & mean & SCA & mean & SCA & mean & SCA & mean & SCA & mean & SCA & mean & SCA & mean \\
\hline $\begin{array}{l}\text { 18.CML } 481 \mathrm{X} \\
\text { CLO2450 }\end{array}$ & 2.06 & 93 & 1.76 & 95 & -5.31 & 172 & 6.04 & 84 & -1.21 & 15 & -1.86 & 31 & $54.90^{* *}$ & 365 & -0.87 & 8 \\
\hline $\begin{array}{l}\text { 19.CML } 496 \text { X } \\
\text { CLO2450 }\end{array}$ & -0.8 & 87 & -0.76 & 90 & 11.81 & 152 & 3.71 & 77 & -0.04 & 15 & -1.14 & 34 & $22.60^{* *}$ & 330 & -1.09 & 6 \\
\hline $\begin{array}{l}\text { 20.CML } 433 \text { X } \\
\text { CLO2450 }\end{array}$ & 0.81 & 89 & 0.76 & 92 & -11.81 & 145 & -3.71 & 73 & 0.04 & 16 & 1.14 & 37 & $-22.60^{* *}$ & 295 & 1.09 & 8.6 \\
\hline $\begin{array}{l}\text { 21.CLG } 1837 \text { X } \\
\text { CML451 }\end{array}$ & $7.94 * *$ & 110 & $8.24 * *$ & 113 & -8.19 & 135 & -5.04 & 50 & $-2.29 * *$ & 12 & -0.89 & 29 & $17.60^{* *}$ & 355 & -0.37 & 7.8 \\
\hline $\begin{array}{l}\text { 22.CL02720 } \\
\text { XCML451 }\end{array}$ & $-7.94 * *$ & 94 & $-8.24 * *$ & 97 & 8.19 & 158 & 5.04 & 64 & $2.29 * *$ & 17 & 0.89 & 32 & $-17.60^{* *}$ & 330 & 0.37 & 8.9 \\
\hline $\begin{array}{l}\text { 23.CML285 X } \\
\text { CML451 }\end{array}$ & 0.69 & 90 & 0.74 & 92 & -3.94 & 163 & -1.79 & 67 & $-2.29 * *$ & 14 & -2.39 & 27 & $22.60^{* *}$ & 345 & $-2.71^{*}$ & 4.6 \\
\hline $\begin{array}{l}\text { 24.CML } 287 \text { X } \\
\text { CML451 }\end{array}$ & -0.6 & 89 & -0.74 & 91 & 3.94 & 163 & 1.79 & 74 & $2.29 * *$ & 19 & 2.39 & 33 & $-22.60^{* *}$ & 310 & $2.71^{*}$ & 10.4 \\
\hline $\begin{array}{l}\text { 25.CML298 X } \\
\text { CML451 }\end{array}$ & -3.06 & 87 & -3.51 & 89 & -10.19 & 128 & -3.79 & 49 & 1.21 & 15 & 1.11 & 28 & $10.10^{*}$ & 290 & 1.17 & 8.7 \\
\hline $\begin{array}{l}\text { 26.CML425 X } \\
\text { CML451 }\end{array}$ & 3.06 & 93 & 3.51 & 97 & 10.19 & 163 & 3.79 & 60 & -1.21 & 13 & -1.11 & 27 & $-10.10^{*}$ & 280 & -1.17 & 6.8 \\
\hline $\begin{array}{l}\text { 27.CML429 X } \\
\text { CML451 }\end{array}$ & 0.19 & 86 & -0.01 & 89 & -2.94 & 139 & -5.54 & 54 & 0.96 & 13 & 1.86 & 28 & -2.4 & 260 & 0.47 & 4.5 \\
\hline $\begin{array}{l}\text { 28.CML430 X } \\
\text { CML451 }\end{array}$ & -0.19 & 86 & 0.01 & 89 & 2.94 & 153 & 5.54 & 68 & -0.96 & 11 & -1.86 & 26 & 2.4 & 275 & -0.47 & 3.9 \\
\hline $\begin{array}{l}\text { 29.CML431 X } \\
\text { CML451 }\end{array}$ & -0.56 & 88 & -0.51 & 91 & 7.06 & 170 & 9.96 & 74 & $1.96^{* *}$ & 17 & 1.86 & 36 & $-17.40^{* *}$ & 310 & -0.43 & 7.4 \\
\hline $\begin{array}{l}\text { 30.CML486 X } \\
\text { CML451 }\end{array}$ & 0.56 & 89 & 0.51 & 92 & -7.06 & 169 & -9.96 & 57 & $-1.96^{* *}$ & 13 & -1.86 & 34 & $17.40^{* *}$ & 355 & 0.43 & 8.6 \\
\hline $\begin{array}{l}\text { 31.CML223 X } \\
\text { CML451 }\end{array}$ & 0.19 & 88 & 0.49 & 91 & 9.06 & 161 & 4.46 & 64 & -0.29 & 14 & -1.14 & 25 & 2.6 & 245 & 1 & 7.1 \\
\hline \multicolumn{17}{|l|}{ Table 4 cont'd } \\
\hline \multirow[t]{2}{*}{ Parents } & \multicolumn{2}{|l|}{ DPS (day) } & \multicolumn{2}{|l|}{ DS (day) } & \multicolumn{2}{|l|}{ PH (cm) } & \multicolumn{2}{|l|}{ EH (cm) } & \multicolumn{2}{|l|}{ EL (cm) } & \multicolumn{2}{|c|}{ NGPR (no.) } & \multicolumn{2}{|l|}{ TGW (gm) } & \multicolumn{2}{|l|}{$Y(t / h a)$} \\
\hline & SCA & mean & SCA & mean & SCA & mean & SCA & mean & SCA & mean & SCA & mean & SCA & mean & SCA & mean \\
\hline $\begin{array}{l}\text { 32.BIL79 X } \\
\text { CML451 }\end{array}$ & -0.19 & 88 & -0.49 & 91 & -9.06 & 153 & -4.46 & 59 & 0.29 & 15 & 1.14 & 29 & -2.6 & 250 & -1 & 5.5 \\
\hline $\begin{array}{l}\text { 33.BIL77 X } \\
\text { CML451 }\end{array}$ & 2.94 & 93 & 2.74 & 96 & -4.19 & 154 & -2.79 & 55 & 1.46 & 16 & 3.61 & 30 & $-86.90^{* *}$ & 141 & -0.13 & 6.2 \\
\hline $\begin{array}{l}\text { 34.CML427 X } \\
\text { CML451 }\end{array}$ & -2.94 & 88 & -2.74 & 91 & 4.19 & 153 & 2.79 & 64 & -1.46 & 13 & -3.61 & 24 & $86.90^{* *}$ & 325 & 0.13 & 6.8 \\
\hline $\begin{array}{l}\text { 35.CML473 X } \\
\text { CML451 }\end{array}$ & 2.94 & 107 & 2.74 & 110 & -5.94 & 142 & 0.96 & 62 & -0.54 & 15 & -0.64 & 29 & $22.60^{* *}$ & 340 & -0.32 & 8.3 \\
\hline $\begin{array}{l}\text { 36.VL1016220 X } \\
\text { CML } 451\end{array}$ & -2.94 & 102 & -2.74 & 105 & 5.94 & 149 & -0.96 & 63 & 0.54 & 16 & 0.64 & 32 & $-22.60^{* *}$ & 305 & 0.32 & 9.3 \\
\hline $\begin{array}{l}\text { 37.CML } 465 \text { X } \\
\text { CML451 }\end{array}$ & -0.81 & 86 & -0.76 & 89 & 4.81 & 157 & 3.96 & 73 & -0.29 & 14 & 2.11 & 36 & $37.60^{* *}$ & 295 & -0.59 & 7.6 \\
\hline $\begin{array}{l}\text { 38.CML } 481 \text { X } \\
\text { CML451 }\end{array}$ & 0.81 & 88 & 0.76 & 91 & -4.81 & 170 & -3.96 & 69 & 0.29 & 15 & -2.11 & 33 & $-37.60^{* *}$ & 230 & 0.59 & 9.1 \\
\hline $\begin{array}{l}\text { 39.CML } 496 \text { X } \\
\text { CML451 }\end{array}$ & -2.31 & 87 & -2.26 & 90 & 7.56 & 163 & 3.96 & 75 & -0.54 & 15 & -2.14 & 30 & $37.60 * *$ & 365 & -0.36 & 8.9 \\
\hline $\begin{array}{l}\text { 40.CML } 433 \text { X } \\
\text { CML } 451\end{array}$ & 2.31 & 92 & 2.26 & 95 & -7.56 & 160 & -3.96 & 70 & 0.54 & 17 & 2.14 & 35 & $-37.60^{* *}$ & 300 & 0.36 & 9.9 \\
\hline SE (Sij) & 3.28 & & 3.33 & & 8.08 & & 8.19 & & 0.7 & & 2.26 & & 4.62 & & 1.28 & \\
\hline SE (Sij-Skl) & 4.62 & & 4.71 & & 11.43 & & 11.59 & & 0.99 & & 3.19 & & 29.45 & & 1.81 & \\
\hline
\end{tabular}

*Significant at $5 \%$ level, ** Significant at I\% level

DPS, days to pollen shedding; DS, days to silk; PH, plant height; EH, ear height; EL, ear length; NGPR, number of grains per row; TGW, I000- grain weight, Y, yield

\section{Heterosis}

The standard/economic heterosis expressed by the $\mathrm{F}_{1}$ hybrids over the standard check variety BHM9 (BARI Hybrid Maize 9) for different characters are shown in Table 5. The measurement of heterosis is different for eight characters in all crosses. Considering commercial hybrid BHM9 as a check, the heterosis ranged from $-1.8 \%$ to $21.0 \%$ for days to pollen shedding and most of the crosses showed significant and positive heterosis except three viz.VL1016220xCLO2450 (-1.8\%), CML429xCML451 (-1.2\%),CML430xCML451(-1.3\%) which exhibited significant and negative heterosis. For days to silking, heterosis ranged from $-2.3 \%$ to $20.4 \%$ and four crosses showed significant and negative heterosis, and only one cross i.e. VL1016220 x CLO2450 showed significant and negative heterosis for 
both days to pollen shedding $(-1.8 \%)$ and silking $(-2.3 \%)$ indicating earliness of the hybrid. Heterosis ranged from plant height and ear height was $-48 \%$ to $-1.4 \%$ and $-46.9 \%$ to $23.8 \%$, respectively. None of the crosses showed significant and positive heterosis over the check BHM9. So there is an enough scope for developing dwarf hybrids. Standard heterosis ranged from $-63.6 \%$ to $2.7 \%,-53.2$ to $11.1 \%$, and -144.7 to $6.8 \%$ for ear length, no of grain per row and thousand grain weight, respectively. These are the yield contributing characters and significant positive heterosis is desirable. Positive heterosis is desirable for grain yield and the heterosis ranged from $158.0 \%$ to $11.6 \%$ which indicated sufficient variability present among the studied hybrids. ${ }^{16}$ And one cross CML431xCLO2450 (11.6\%*) identified as highest significant positive heterosis and two crosses showed positive hetrerosisVL1016220 x CLO2450 (3.2\%) and CML287xCML451(2.4\%). Matin and Malik et al., ${ }^{16,17}$ found -56.59 to $15.60 \%$ standard heterosis for yield in their study.

Table 5 Heterosis of the crosses over BHM9 for different characters in maize

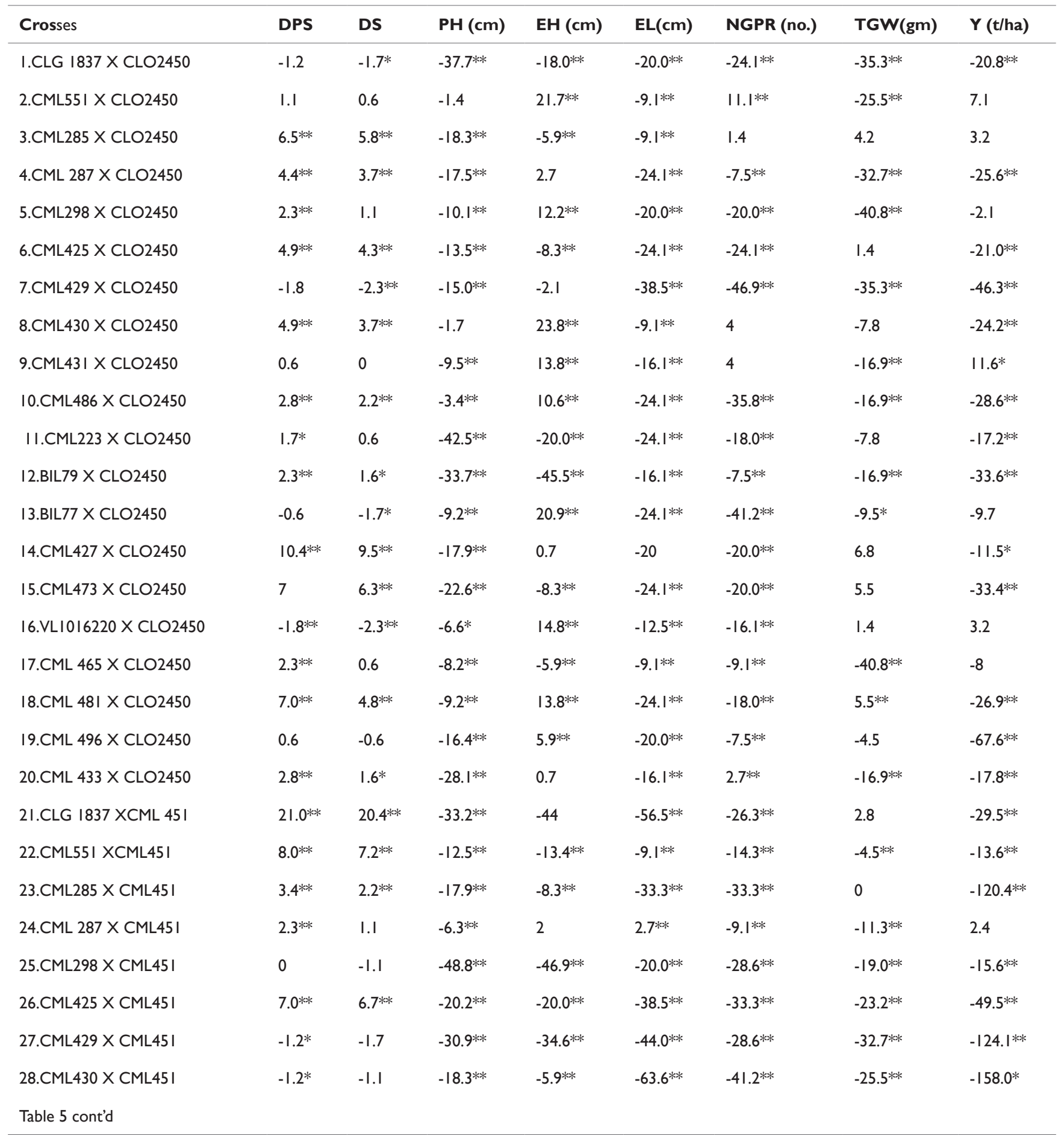


Table Continued

\begin{tabular}{|c|c|c|c|c|c|c|c|c|}
\hline Crosses & DPS & DS & $\begin{array}{l}\mathrm{PH} \\
(\mathrm{cm})\end{array}$ & $\begin{array}{l}\mathrm{EH} \\
(\mathrm{cm})\end{array}$ & $\begin{array}{l}\text { EL } \\
(\mathrm{cm})\end{array}$ & NGPR (no.) & $\begin{array}{l}\text { TGW } \\
\text { (gm) }\end{array}$ & $\begin{array}{l}Y \\
\text { (t/ha) }\end{array}$ \\
\hline 30.CML486 X CML45I & $2.8 * *$ & $2.2^{* *}$ & $-13.2 * *$ & $-26.3^{* *}$ & $-38.5^{* *}$ & $-7.5^{* *}$ & 2.8 & $-17 . \mid * *$ \\
\hline 31.CML223 X CML45I & I.I & I.I & $-17.1 * *$ & $-12.5 * *$ & $-33.3 * *$ & $-44.0^{* * *}$ & $-40.8 * *$ & $-41.5 * *$ \\
\hline 32.BIL79 X CML45I & I.I & 0.6 & $-24.2^{* *}$ & $-23.1 * *$ & $-24.1 * *$ & $-26.3^{* *}$ & $-38.0 * *$ & $-83.7 * *$ \\
\hline 33.BIL77 X CML45I & $7.0 * *$ & $6.3^{* *}$ & $-38.2^{* *}$ & -30.9 & $-16.1 * *$ & $-22.0 * *$ & $-144.7 * *$ & $-63.7 * *$ \\
\hline 34.CML427 X CML45I & I.I & I.I & $-22.2^{* *}$ & $-12.5 * *$ & $-38.5^{* *}$ & $-53.2 * *$ & -6.2 & $-48.7 * *$ \\
\hline 35.CML473 X CML45I & $19.2^{* *}$ & $17.8^{* *}$ & $-33.7 * *$ & $-17.1 * *$ & $-24.1 * *$ & $-24 . I^{* *}$ & -1.5 & $-22.0 * *$ \\
\hline 36.VLI0I6220 X CM 45I & $14.8 * *$ & $13.9 * *$ & $-16.0^{* *}$ & $-14.3^{* *}$ & $-12.5^{* *}$ & $-14.3^{* *}$ & $-13.1 * *$ & -9 \\
\hline 37.CML 465 X CML45I & -0.6 & -1.1 & $-9.8 * *$ & 1.4 & $-28.6 * *$ & -1.4 & $-16.9 * *$ & $-33.7 * *$ \\
\hline 38.CML 48I X CML45I & $1.7 *$ & I.I & $-10.1 * *$ & -5.1 & $-20.0 * *$ & $-10.8 * *$ & $-50.0 * *$ & -||$. \mid * *$ \\
\hline 39.CML 496 X CML45 I & 0 & -0.6 & $-9.8 * *$ & 3.4 & $-20.0 * *$ & $-22.0 * *$ & 5.5 & $-13.9 * *$ \\
\hline 40.CML 433 X CML45 I & $5.5^{* *}$ & $4.8 * *$ & $-13.9 * *$ & -2.9 & $-9.1 * *$ & -2.9 & $-15.0 * *$ & -1.5 \\
\hline Mean & 3 & -18 & -7 & -23 & -18 & -18 & $-31.5^{* *}$ & 3 \\
\hline Min & -1.8 & -2.3 & -48.8 & -46.9 & -63.6 & -53.2 & -145 & -158 \\
\hline $\operatorname{Max}$ & 21 & 20.4 & -1.4 & 23.8 & 2.7 & II.I & 6.8 & 11.6 \\
\hline SE & 0.82 & 0.81 & 1.82 & 2.83 & 2.11 & 2.44 & 4.09 & 5.74 \\
\hline $\mathrm{CD}(0.0 \mathrm{I})$ & 2.22 & 2.18 & 4.94 & 7.65 & 5.7 & 6.6 & 11.07 & 15.55 \\
\hline
\end{tabular}

\section{Conclusion}

It can be concluded that the lines CML430, CML429 and CML223 were found good general combining ability effects for yield and important yield contributing characters. For earliness and dwarfness the lines CML427 and BIL77 showed expected general combining ability effects, respectively. And the tester CML451 showed significant positive GCA indicating good combiner for yield. However, these results suggest that these inbred lines have the potentiality for producing superior single crosses. Two crosses, CML287xCML451 had significant positive specific combining ability effects for grain yield and the cross CML431xCLO2450 showed highest mean value along with significant positive heterosis for grain yield were selected and could be advanced for commercial hybrids development after verifying the performance in over locations.

\section{Acknowledgements}

The authors are thankful to CIMMYT for providing inbreds for this research.

\section{Conflict of interest}

The author declares there is no conflict of interest.

\section{References}

1. Pingali PL. CIMMYT 1999-2000 World Maize Facts and Trends. Meeting World Maize Needs: Technological Opportunities and Priorities for the Public Sector. Mexico; CIMMYT. 2001;60.

2. Kempthorne O. An introduction to genetic statistics. New York; John Wiley and Sons, Inc. London: Chapman and Hall Limited; 1957.

3. Patteraon HD, Williams ER. A new clas. Of resolvable block design. Biometrika. 1976;63:83-12.

4. Singh RK, Chaudhary BD. Biometrical Methods in Quantitative Genetics Analysis. Kalyani Publishers, New Delhi, India. 1999. 318 p.

5. Yazachew GE, Pangirayi BT, Beatrice EI. General And Specific Combining Ability Studies Of Selected Tropical White Maize Inbred Lines For Yield And Yield Related Traits. Int J of Agri Sci and Res. 2017;7(2):381-396

6. Kanagarasu S, Nallathambi G, Ganesan KN. Combining ability analysis for yield and its component traits in maize (Zea mays L.). Electronic $J \mathrm{Pl}$ Breed. 2010;1(4):915-920.

7. Kumar GP, Narsimha VR, Sudheer SK, et al. Combining ability studies in newly developed inbred lines in maize (Zea mays L.). Intl J of Pl Ani And Env Sci. 2014;4(4):229-234. 
8. Ali G, Ahmed I, Dar SA, et al. Combining ability analysis for yield and its component traits in high altitude maize (Zea mays L.) inbreds. Advances in life sciences. 2012;1(1):66-69.

9. Kumar BS, Prakash M, Sathyanarayanan G. Studies on combining ability and heterosis through line $\mathrm{x}$ tester analysis in maize (Zea mays L.). Crop Research (Hisar). 2012;43(1/2/3):153-157.

10. Amiruzzaman M. Exploitation of hybrid vigour from normal and quality protein maize. Ph. D. Dissertation. Department of Genetics and Plant Breeding. Bangladesh Agricultural University. 2010. 50-111 p.

11. Bhavana P, Singh RP, Gadag RN. Gene action and heterosis for yield and yield components in maize (Zea mays). Indian $J$ of Agril Sci. 2011;81(2):163-166.

12. Jawaharlal J, Reddy GL, Kumar RS.. Heterosis for yield component traits in maize (Zea mays L.). Indian J of Agril Res. 2012;46(2):184-187.
13. Amin MN, Amiruzzaman M, Ahmed A. Evaluation of Inbred Lines of Maize (Zea mays L.) through Line x Tester Method. Bangladesh $J$ of Agril Res. 2014;39(4):675-683.

14. Ivy NA, Howlader MS. Combining ability in maize. Bangladesh J Agril Res. 2000;25:385-392.

15. Nadarajan N, Gunasekaran M. Quantitative genetics and biometrical techniques in plant breeding. Kalyani Publishers, India, 2008. 108 p.

16. Matin MQI, Rasul MG, Islam AKMA, et al. Combining Ability and Heterosis in Maize (Zea mays L.). American J of Bio Sci. 2016;4(6):84 90 .

17. Malik SI, Malik HN, Minhas NM. General and Specific combining ability studies in maize. Int J Agri Biol. 2004;6:856-859. 